\title{
CONSERVACIÓN DE PULPA DE CARAMBOLA (Averrhoa Carambola) CON APLICACIÓN DE MÉTODOS DE FACTORES COMBINADOS
}

\section{CONSERVATION OF PULP CARAMBOLA (AVERRHOA CARAMBOLA) WITH APPLICATION OF METHODS OF FACTORS COMBINED}

\author{
Edgar Rafael Acosta López² y Angélica Castro Garay \\ Facultad de Ciencias Agrarias - Satipo
}

\section{RESUMEN}

El objetivo del presente trabajo fue estudiar la estabilidad físico-química, sensorial y microbiológica durante el almacenamiento a temperatura ambiente de pulpa de carambola. Se han realizado estudios de aplicación de la tecnología de factores combinados en la conservación de pulpa de carambola, para que puedan ser empleados como materia prima para la obtención de productos finales como jalea, mermelada, jugo, helado, paleta congelada, néctar y dulces 0 postres de sobremesa. Se ha empleado la acción de diversos factores de barreras: reducción de la aw, preservante (SK) y pasteurización $\left(89^{\circ} \mathrm{Cx} 15\right.$ segundos y $80^{\circ} \mathrm{C}$ $x 5$ minutos). Los factores de estudio fueron la pasteurización, la relación de concentración de pulpa/concentración de soluto (CP/CS; 1/1/2 y 1/1/4) como depresor de aw se ha utilizado azúcar refinado grado alimentario y el sorbato de potasio SK $(0,2 \%$ y $0,4 \%)$. Se ha mantenido el $\mathrm{pH}$ de la pulpa de carambola en equilibrio y se ha considerado como el tratamiento óptimo, aquel que mantiene inalterable el color característico de la pulpa de carambola (amarillo anaranjado) durante el tratamiento y un tiempo de vida útil de 60 días aproximadamente en condiciones normales de temperatura y empacados en envases de plástico con tapa. La evolución de sólidos solubles, $\mathrm{pH}$ y acidez no mostraron diferencias significativas $(=0.05)$ durante los 60 días de almacenamiento. El crecimiento microbiano reporta valores de diferencias no significativas con respecto al tiempo de almacenamiento. Desde el punto de vista sensorial y como una característica fundamental de calidad, el color de la pulpa de carambola denota variación notable del color natural a partir de los 45 días de almacenamiento, siendo esta característica la que orienta el tiempo de vida útil de esta pulpa.

Palabras clave: carambola; averrhoa carambola; métodos combinados; frutas tropicales; conservación de frutas.

\section{ABSTRACT}

The objective of the present work was to study the physical-chemistry, sensorial and microbiological stability during the storage to environmental temperature of carom pulp. Studies of technological application of factors combined in the conservation of carom pulp have been carried out, so that they can be used as raw material for the obtaining of end products like jelly, marmalade, juice, ice cream, frozen palette, nectar and sweet or after-dinner desserts. The action of diverse factors of barriers has been used: reduction of the aw, preservers (SK) and pasteurization $\left(89^{\circ} \mathrm{Cx} 15\right.$ seconds and $80^{\circ} \mathrm{C} \times 5$ minutes). The study factors were the pasteurization, and for the relationship of concentration of soluto pulpa/concentration (CP/CS; $1 / 1 / 2$ and 1/1/4) as aw depressor, refined

\footnotetext{
2 edgaracosta30@hotmail.com
} 
sugar alimentary grade and the sorbate of potassium SK $(0,2 \%$ and $0,4 \%)$ has been used. The $\mathrm{pH}$ of the carom pulp has been maintained in balance and it has been considered as the good treatment, and that maintains unalterable the characteristic color of the carom pulp (yellow orange) during the treatment and also a time of useful life of 60 days approximately under normal conditions of temperature and packed in containers of plastic with cover. The evolution of soluble solids, $\mathrm{pH}$ and acidity didn't show significant differences $(=0.05)$ during the 60 days of storage. The microbial growth reports values of non significant differences with regard to the time of storage. From the sensorial point of view and like a fundamental characteristic of quality, the color of the carom pulp denotes remarkable variation of the natural color starting from the 45 days of storage, being this characteristic the one that guides the time of useful life of this pulp.

Key word: averrhoa carambola, combined methods, tropical fruits, conservation of Fruits

\section{INTRODUCCIÓN}

En diversos lugares de la provincia de Satipo crece la carambola y es consumido en refrescos y ensaladas por los pobladores, no obstante en la época de abundancia se desperdician cantidades considerables, ya que la producción excede la demanda local y regional. Una opción para evitar el desperdicio, además de otorgar un valor agregado al fruto es procesarlo y comercializarlo. (Acosta, 2009).

La obtención de pulpas a partir de frutos es un proceso ventajoso por la versatilidad de usos que puedan dar las mismas; sin embargo deben considerarse las condiciones que permitan conservar la pulpa por el mayor tiempo posible. La tecnología de métodos combinados ofrece varias ventajas como son: el empleo de tecnología y equipo sencillo y no costoso, conserva las propiedades del producto fresco aplicable a zonas rurales en donde se pueden conservar los frutos in situ y por medio de este método se han obtenido productos estables a temperatura ambiente. (Hernández, Barrera; 2004).

Con la tecnología de los métodos combinados, se pretenden alcanzar niveles de humedad intermedia $(\mathrm{HI})$. El proceso aplicado a frutas se basa en la combinación de un tratamiento térmico suave, una ligera reducción de la actividad de agua, descenso del pH y adición de niveles permitidos de sustancias químicas, en un proceso de estabilización por equilibrio de los trozos de frutas en el sistema jarabe-ácido-preservantes en el caso de conservación en trozos, y en un sistema pulpa-prersevante-soluto-ácido en el caso de pulpas.

Utilizando los métodos combinados se podrá conservar la pulpa de carambola al medio ambiente y se tendrá un producto intermedio para que pueda ser empleado como materia prima para la obtención de productos finales como jalea, mermelada, jugo, helado, paleta congelada, granizado, néctar y dulces o postres de sobremesa.

En consecuencia con lo planteado la investigación pretende conservar la pulpa pasteurizada de carambola a temperatura ambiente utilizando métodos combinados.

En la investigación se formula el siguiente problema ¿cuál de los métodos combinados permitirá mantener una buena calidad microbiana (mohos y levaduras) al almacenar la pulpa del fruto de carambola a temperatura ambiente? Del cual se deduce la siguiente hipótesis: La aplicación de métodos combinados permitirá mantener una buena calidad microbiana (mohos y levaduras) al almacenar la pulpa de carambola a temperatura ambiente.

El objetivo general de la investigación fue, estudiar la estabilidad físico-química y microbiológica 
durante el almacenamiento a temperatura ambiente de la pulpa de carambola.

\section{MATERIAL Y MÉTODOS}

La investigación se realizó en el Centro de Producción de la Escuela Académico Profesional de Ingeniería en Industrias Alimentarias Tropical de la Facultad de Ciencias Agraria de la Universidad Nacional del Centro del Perú. La Unidad experimental fue de $50 \mathrm{~g}$ de pulpa de carambola pasteurizada, envasada y almacenada a temperatura ambiente en un lugar fresco a $25^{\circ} \mathrm{C}$.

Se utilizó como insumos azúcar blanca refinada y como conservador sorbato de potasio.

Se realizó los análisis físico-químicos y microbiológicos de la pulpa: $\mathrm{pH}$, potenciométricamente con un ph-metro ORION modelo S-350; sólidos solubles con un refractómetro calibrado térmicamente marca Abbe; contenido de agua por método AOAC; acidez, mohos, levaduras por Petrifilm (2002). La metodología experimental consistió en la obtención de la pulpa y la aplicación de métodos combinados a la pulpa del fruto de carambola, Los factores que se probaron fueron los siguientes: depresor de actividad de agua, se añade azúcar a la pulpa en dos niveles teniendo en cuenta la relación pulpa /soluto $(1 / 0,5)$ y $(1 / 0,25)$ como soluto se utilizó sacarosa (azúcar Blanca refinada), sorbato de potasio (SK) $0,2 \%$ y $0,4 \%$, tratamiento térmico a la pulpa a $80^{\circ} \mathrm{C}$ por 5 minutos y $89^{\circ} \mathrm{C}$ por 15 segundos. Al combinar estos factores con sus respectivos niveles se obtuvieron 8 tratamientos

Análisis estadístico: para la caracterización de la pulpa se realizaron dos repeticiones con tres réplicas para cada una de las determinaciones, reportándose los promedios y desviaciones estándar. Para la aplicación de métodos combinados se tuvo como unidad experimental
$50 \mathrm{~g}$ de pulpa envasada y almacenada a 25 +- $1,0^{\circ} \mathrm{C}$. Se utilizó un diseño factorial 23 completamente aleatorizado, con tres replicas independientes, dando como resultado un total de 24 muestras para cada tiempo, las cuales fueron bloqueadas por día, es decir, las ocho muestras de combinaciones de tratamientos fueron analizadas en un día y las otras dos replicas en los consecutivos días existiendo un día de diferencia entre ellas. El análisis estadístico de los resultados obtenidos se hizo con un análisis de varianza para tres factores (ANOVA). La variable de respuesta que se seleccionó para conocer el efecto de los factores en los tratamientos probados fue la cuantificación de hongos y levaduras.

\section{RESULTADOS}

El rendimiento de la pulpa de carambola refinada fue de $74,7 \%$.

Resultados fisicoquímicos de la pulpa de carambola refinada fresca

Cuadro 1. Resultados fisicoquímicos de la pulpa de carambola sin la aplicación de métodos combinados.

\begin{tabular}{cc}
\hline Análisis fisicoquímico & Resultado \\
\hline $\mathrm{pH}\left(\right.$ a una $\left.\mathrm{T}=20^{\circ} \mathrm{C}\right)$ & 2,3 \\
Densidad $\left(20^{\circ} \mathrm{C}\right) \mathrm{g} / \mathrm{mL}$ & 1,023 \\
Acidez cítrica $(\%)$ & 0,74 \\
Sólidos solubles ${ }^{\circ}$ Brix & 6,30 \\
\hline
\end{tabular}

\section{Resultados fisicoquímicos, microbiológicos y sensoriales de la pulpa de carambola por métodos combinados}

La pulpa calentada, estabilizada y empacada se almacenó durante 2 meses a temperatura 
ambiente $\left(25^{\circ} \mathrm{C}\right)$, habiéndose realizado controles ambiente no denotan diferencias significativas fisicoquímicos, microbiológicos y sensoriales $(a=0.05)$ respecto al tiempo de almacenamiento, durante este tiempo. El pH, sólidos solubles y la los cuales se pueden observar en los cuadros 2, acidez durante el almacenamiento a temperatura 3 y 4.

Cuadro 2. Análisis de pH de la pulpa de carambola conservada por métodos combinados

\begin{tabular}{clllllllll}
\hline \multirow{2}{*}{ Días } & \multicolumn{10}{c}{ Tratamiento } \\
\cline { 2 - 10 } & Control & 1 & 2 & 3 & 4 & 5 & 6 & 7 & 8 \\
\hline 0 & 2,3 & 2,3 & 2,45 & 2,35 & 2,4 & 2,42 & 2,3 & 2,31 & 2,33 \\
15 & 2,9 & 2,4 & 2,55 & 2,4 & 2,4 & 2,42 & 2,32 & 2,34 & 2,4 \\
30 & 3,1 & 2,4 & 2,55 & 2,45 & 2,42 & 2,42 & 2,32 & 2.36 & 2,4 \\
45 & 3,3 & 2,4 & 2,6 & 2,5 & 2,42 & 2,46 & 2,35 & 2,36 & 2,42 \\
60 & & 2,5 & 2,6 & 2,5 & 2,45 & 2,46 & 2,35 & 2,38 & 2,42 \\
\hline
\end{tabular}

Cuadro 3. Análisis de \% Acidez de la pulpa de carambola conservada por métodos combinados

\begin{tabular}{clllllllll}
\hline \multirow{2}{*}{ Días } & \multicolumn{10}{c}{ Tratamiento } \\
\cline { 2 - 10 } & Control & 1 & 2 & 3 & 4 & 5 & 6 & 7 & 8 \\
\hline 0 & 0,74 & 0,735 & 0,73 & 0,73 & 0,72 & 0,73 & 0,73 & 0,72 & 0,71 \\
15 & - & 0,72 & 0,71 & 0,71 & 0,71 & 0,70 & 0,72 & 0,72 & 0,70 \\
30 & & 0,71 & 0,70 & 0,72 & 0,71 & 0,69 & 0,72 & 0,71 & 0,70 \\
45 & 0,70 & 0,69 & 0,70 & 0,70 & 0,69 & 0,71 & 0,71 & 0,70 \\
60 & 0,70 & 0,68 & 0,70 & 0,69 & 0,69 & 0,71 & 0,71 & 0,69 \\
\hline
\end{tabular}

Cuadro 4. Análisis de sólidos solubles ( ${ }^{\circ}$ Brix) de la pulpa de carambola conservada por métodos combinados

\begin{tabular}{llllllllll}
\hline \multirow{2}{*}{ Días } & \multicolumn{8}{c}{ Tratamiento } \\
\cline { 2 - 10 } & Control & 1 & 2 & 3 & 4 & 5 & 6 & 7 & 8 \\
\hline 0 & 6,30 & 6,45 & 6,43 & 6.5 & 6.5 & 6.4 & 6.45 & 6.4 & 6.4 \\
15 & 5,80 & 6,2 & 6,35 & 6.4 & 6.4 & 6.35 & 6.3 & 6.35 & 6.35 \\
30 & & 6,1 & 6,2 & 6.3 & 6.4 & 6.3 & 6.25 & 6.3 & 6.3 \\
45 & & 6,0 & 6,1 & 6.1 & 6.2 & 6.25 & 6.2 & 6.3 & 6.2 \\
60 & & 6,0 & 6,1 & 6.1 & 6.2 & 6.25 & 6.1 & 6.2 & 6.0 \\
\hline
\end{tabular}

Antes de describir el comportamiento de los 102 ufc/g según las normas existentes (NTS.

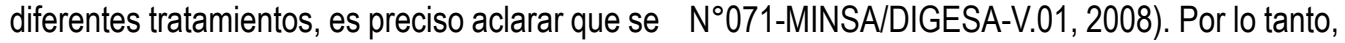
ha tomado como limite admisible para continuar todos aquellos tratamientos donde rebasaron con el monitoreo de los tratamientos probados un estos valores se eliminaron. valor máximo de mohos de 103 ufc/g y levaduras 
Cuadro 5. Cuantificación de mohos (ufc/g) en los tratamientos durante el almacenamiento a $25^{\circ} \mathrm{C}$

\begin{tabular}{llllllllll}
\hline Días & \multicolumn{10}{c}{ Tratamiento } \\
\cline { 2 - 11 } & Control & 1 & 2 & 3 & 4 & 5 & 6 & 7 & 8 \\
\hline 0 & 10 & 10 & 8 & 7 & 11 & 12 & 10 & 0 & 0 \\
15 & 1100 & 120 & 170 & 13 & 14 & 14 & 17 & 0 & 0 \\
30 & & 200 & 220 & 15 & 18 & 28 & 29 & 0 & 0 \\
45 & & 280 & 300 & 34 & 48 & 36 & 38 & 0 & 0 \\
60 & & 430 & 450 & 60 & 72 & 69 & 66 & 0 & 0 \\
\hline
\end{tabular}

Cuadro 6. Cuantificación de levaduras (ufc/g) en los tratamientos durante el almacenamiento a $25^{\circ} \mathrm{C}$

\begin{tabular}{llllllllll}
\hline Días & \multicolumn{10}{c}{ Tratamiento } \\
\cline { 2 - 9 } & Control & 1 & 2 & 3 & 4 & 5 & 6 & 7 & 8 \\
\hline 0 & 25 & $<10$ & $<10$ & $<10$ & 11 & 8 & $<10$ & $<10$ & $<10$ \\
15 & 500 & $<10$ & 20 & $<10$ & 14 & 10 & 15 & $<10$ & 16 \\
30 & ND & $<10$ & 45 & 30 & 18 & 20 & 30 & 24 & 27 \\
45 & & 30 & 40 & 50 & 48 & 42 & 40 & 39 & 41 \\
60 & & $<100$ & $<100$ & $<100$ & 72 & 50 & 68 & 70 & 66 \\
\hline
\end{tabular}

ND: no determinado

$\mathrm{Ufc} / \mathrm{mL}=$ Unidades formadoras de colonias por gramo

En cuanto a las experiencias desarrolladas, después del procesado, es decir al día cero de almacenamiento, los recuentos mohos fueron máximo de $10 \mathrm{ufc} / \mathrm{g}$ y para los procesados con $\left(89^{\circ} \mathrm{C} \times 15 \mathrm{~s}\right)$ y con una concentración de SK de $0,2 \%$ y $0,4 \%$ no se encontró mohos, resultados que son los esperados, puesto que a mayor temperatura mayor disminución de la tasa microbiana en la pulpa; sin embargo, los valores obtenidos son aceptables. Haciendo un seguimiento durante el almacenamiento, conforme se muestra en el Cuadro 5 , los niveles de crecimiento de mohos no son elevados, pues en ningún caso pasan del límite establecido (103 ufc/g). Durante los 60 días de almacenamiento el crecimiento microbiano no llegó a valores en la que microbiológicamente se pueda desechar las muestras tanto desde el punto de recuento de mohos y levaduras. La aplicación de métodos combinados permite la conservación de los mismos a temperatura ambiente y se mantiene la seguridad microbiológica.

La prueba de puntos y calificación se desarrolló al inicio y durante el almacenamiento de 60 días, respecto al color, sabor y aroma. El color amarillo anaranjado de la pulpa de carambola mantiene su color natural después del procesamiento, con las pulpas que se adicionaron menos sacarosa $(1 / 0.25)$ y que se aplicaron menor temperatura de pasteurización $\left(80^{\circ} \mathrm{C} \times 5\right.$ minutos). Esto podría deberse a que durante el calentamiento y posterior enfriamiento lento, ocurren reacciones de oscurecimiento y caramelización de los azúcares.

Durante el almacenamiento las evaluaciones sensoriales indican que a partir de 45 días las pulpas procesadas con $89^{\circ} \mathrm{C} \times 15$ segundos con relación a la pulpa soluto de $1 / 0.25$, encuentran diferencias significativas a un nivel de $95 \%$ de confianza con respecto al color inicial de almacenamiento. 
La aplicación de $89^{\circ} \mathrm{C}$ x 15 seg.y relación pulpa/soluto de 1/0.25 es considerada como el tratamiento óptimo, ya que en estas condiciones el color característico de la pulpa de carambola se mantiene casi inalterable.

Los otros tratamientos $\left(1 / 0.5 ; 80^{\circ} \mathrm{C} \times 5 \mathrm{~min}\right.$; $0,2 \% \mathrm{SK}),\left(1 / 0.5 ; 80^{\circ} \mathrm{Cx} 5 \mathrm{~min} ; 0,4 \% \mathrm{SK}\right)$, inician el almacenamiento con el color de la pulpa ligeramente modificada, lo que hace que el tiempo de almacenamiento desde el punto de vista del color sea muy pequeño.

En cuanto al aroma, el análisis de varianza reporta que hay diferencias significativas con respecto al factor días de almacenamiento en sus efectos simples, sin embargo en sus interacciones dobles de SK y temperatura-tiempo de pasteurización no denota diferencia significativa.

La valoración promedio de los panelistas según la escala aplicada a los 45 días muestra que la pulpa tiene un olor sui géneris débil, y a los 60 días, olor sui géneris muy débil.

\section{DISCUSIÓN}

El valor del rendimiento de la pulpa de carambola obtenida es ligeramente mayor a lo reportado por (ACOSTA, 2009) 71,3\%. VILLA (2008) menciona un rendimiento en pulpa que varía de $60-75 \%$ que depende del estado de madurez; Hernández y Barrera (2004) reportaron un rendimiento para pulpa de carambola en estado verde $65,56 \%$, pintón $68,88 \%$ y maduro $72,87 \%$. El rendimiento obtenido se encuentra dentro de este rango. Las diferencias se pueden atribuir al estado de madurez de la fruta, perdidas durante el pulpeado, tamaño de abertura del tamiz utilizado para la refinación de la pulpa, procedencia y variedad de la fruta.

El pH de la pulpa de carambola fue de 2,3. Acosta (2009) determinó un valor de $\mathrm{pH}$ de 2,4; Barzola (2008) 2,1 y Tello et al; (2002) el valor obtenido se encuentra dentro del rango. La variación puede ser debido a la procedencia y estado de madurez. La cantidad de ácidos totales expresados en ácido cítrico de la pulpa de carambola fue de $0,74 \mathrm{~g}$ de ácido cítrico/100 g (0,75\%). Valores semejantes presentan los reportados por Barzola (2008) $0,737 \%$, Acosta (2009) 0,74\% y (Tello et al., 2002) $0,72 \%$. Los grados Brix obtenidos en la pulpa del fruto de carambola fue de 6,3, valor que se asemeja a lo reportado por Acosta (2009) y ligeramente superior a lo reportado por Barzola (2008) quien determinó un valor de 6,0 y menor a lo reportado por Tello et al., (2002) quien obtuvo un valor $7,2^{\circ} \mathrm{Brix}$.

El análisis estadístico de las características fisicoquímicas de los tratamientos de pulpa pasteurizada de carambola muestra que no existen diferencias significativas en las variables de $\mathrm{pH}$, acidez y ${ }^{\circ}$ Brix. En los 2 meses de almacenamiento.

El deterioro de las pulpas de frutas está asociado, generalmente, con mohos y levaduras, pues su $\mathrm{pH}$ y actividad de agua reducida, limitan el crecimiento de un gran número de bacterias, principalmente patógenas. El SK es un conservador efectivo contra mohos y levaduras, pero si los niveles de adición son muy pocos e insuficientes, puede existir crecimiento de microorganismos. El comportamiento microbiológico de los tratamientos durante los 60 días de almacenamiento a temperatura ambiente $25^{\circ} \mathrm{C}$, se muestra en los Cuadros 5 y 6.

En el día cero de almacenamiento, los recuentos de mohos fueron máximo de 10 ufc/g y para los procesados con $\left(89^{\circ} \mathrm{C} \times 15 \mathrm{~s}\right)$ con una concentración de SK de 0,2\% y 0,4\% no se encontró mohos, resultados que son los esperados, puesto que a mayor temperatura mayor disminución de la tasa microbiana en la pulpa; sin embargo, los valores obtenidos son aceptables. Haciendo un seguimiento durante el almacenamiento, conforme se muestra en el Cuadro 5 , los niveles de crecimiento de mohos 
no son elevados, pues en ningún caso pasan del límite establecido (103 ufc/g). Durante los 60 días de almacenamiento el crecimiento microbiano no llegó a valores en la que microbiológicamente se pueda desechar las muestras tanto desde el punto de recuento de mohos y levaduras. La aplicación de métodos combinados permite la conservación de los mismos a temperatura ambiente y se mantiene la seguridad microbiológica.

El ANVA reporta diferencia significativa del sabor con el factor tiempo de almacenamiento, en el aroma no hay diferencia significativa con la interacción temperatura- tiempo de pasteurización, y la valoración promedio de los panelistas según la escala aplicada a los 45 días mantiene un sabor ligeramente agradable, a los 60 días de almacenamiento la calificación promedio es de un sabor poco agradable.

- El rendimiento óptimo de la pulpa de carambola a nivel de laboratorio fue de $74,7 \%$.

- Las características fisicoquímicas que presentó la pulpa fresca de carambola fueron: pH 2,3; acidez cítrica $0,74 \%$ y sólidos solubles $6,3^{\circ}$ Brix.

- La tecnología de los métodos combinados en la conservación de pulpa de carambola ha dado resultados satisfactorios, logrando incrementar su tiempo de vida útil en condiciones ambientales, empacados en frascos de polietileno con tapa Twist-off, sin la utilización de frío.

- La aplicación de $89^{\circ} \mathrm{Cx} 15$ segundos, relación pulpa/soluto de $1 / 0.25$ y $0,2 \%$ de sorbato de potasio; es considerando como el tratamiento óptimo, considerando que en estas condiciones, el color característico de la pulpa de carambola se mantiene inalterable durante el tratamiento y su tiempo de vida útil es de 45 días aproximadamente en condiciones normales de temperatura y empacados en frascos de polietileno con tapa Twist-off.
- Durante los 60 días de almacenamiento el crecimiento microbiano no llegó a valores en los que microbiológicamente se pueda desechar las muestras desde el punto de recuento de mohos y levaduras. .

- El pH, los sólidos solubles y la acidez durante el almacenamiento a temperatura ambiente, no denotan diferencias significativas respecto al tiempo de almacenamiento.

\section{REFERENCIAS BIBLIOGRÁFICAS}

Acosta L., E.R. Influencia de la temperatura y concentración en las propiedades reológicas de la pulpa de carambola. [Tesis post grado]. Escuela de Post Grado UNCP. Huancayo-Perú. 2009.

Alzamora S. M. Alimentos conservados por factores combinados. Ed. Aguilera. Temas en tecnología de alimentos. p 45. CYTED vol. 1. Instituto Politécnico Nacional. México. 1997.

Barbosa C. G. V, Pothakamuy R. Usha, P. E. Conservación no térmica de alimentos. P.293. Acribia S.A. España. 1999.

Cerezal, P. y Duarte, G. Sensory influence of chemical additives in peeled cactus pears (Opuntia ficus indica L. Miller) in sirup conserved by combined methods. J: PACD 102; 109. 2004.

García R. y Reátegui, M. Conservación de Pulpa de Aguaje por métodos combinados. Revista Amazónica de Investigación Alimentaria RAIA. V.2. $N^{\circ} 1$, p. 59-68 (2002). Facultad de Ingeniería en Industrias Alimentarias de la UNAP, Iquitos, Perú. 2002.

Hernández, G., María Soledad; Barrera G. Jaime Alberto. Bases técnicas para el aprovechamiento agroindustrial de especies nativas de la Amazonía. Instituto Amazónico de Investigaciones Científicas, SINCHI. Universidad de la Amazonia. Bogotá, Colombia. 100 p. 2004. 
ICMSF. Microorganismos de los alimentos. Su Rees J. y Bettison J. Procesado térmico y significado y métodos de enumeración. 2da ed. envasado de los alimentos. Zaragoza. Acribia. zaragoza. Acribia. 464 P. 2000. 288 p. 1994.

Leistner, Lothar. 2000. Basic aspect of food preservation by hurdle technology. Journal Food Microbiology 55:181-186.

Ordoñez Pereda, Juan. Tecnología de los alimentos. Volumen 1. Componentes de los alimentos y procesos. P 253. SINTESIS. España. 1998

Ray Bideck, Fundamental Food Microbiology, $p$ 46, 62, 66, 68-70. CRC Press Inc. USA. 1996.
Tello, D.W.O. Conservación de (Averrhoa carambola) carambola por azúcar y calor" [Trabajo de fin de carrera] Facultad de Ingeniería en Industrias Alimentarias de la UNAP, Iquitos, Perú. Revista Amazónica de Investigación Alimentaria, v.2, no 1, p. 49 - 58. 2002.

Villanueva Cañongo, Claudia. Caracterización parcial y evaluación de la estabilidad de la pulpa del fruto de Cytocarpa procera conservada por métodos combinados. Tesis para obtener el Título de Ingeniero de Alimentos. Universidad Tecnológica de Mixteca. Huajapan-México. 2006. 